\title{
Bilateral abducens nerve paresis associated with aneurysmal subarachnoid hemorrhage: report of two cases
}

\begin{abstract}
We present the results on two cases of abducens nerve paresis after aneurysmal subarachnoid hemorrhage. Both uncommon finds in this context. The most accepted theory on the origin of this matter is primary compression caused by cisternal clot. Patients presented significant evolution and fully recovered from abducens paresis within the third and sixth month follow up.
\end{abstract}

Keywords: Abducens nerve pareses, Aneurysmal subarachnoid hemorrhage, Prepontine cisternal clot
Volume 2 Issue 6 - 2015

Jaime Lopez Calle

Neurosurgery Section, Arzobispo Loayza Hospital, Peru

Correspondence: Jaime Lopez Calle, Neurosurgery Section, Arzobispo Loayza Hospital, Lima, Peru, Email lopezcallej@gmail.com

Received: July 17, 2015 | Published: September II, 2015
Abbreviations: SAH, Aneurysmal Subarachnoid Hemorrhage; VI CN, abducens Cranial Nerve; CSF, Cerebrospinal Fluid; GCS, Glasgow Coma Scale; CT, Computed Tomography; PComA, Posterior Communicating Artery; mRS, modified Rankin Scale; AComA, Anterior Communicating Artery; PICA, Posterior Inferior Cerebellar Artery

\section{Introduction}

Compromise of the sixth nerve after aneurysmal subarachnoid hemorrhage $(\mathrm{SAH})$ is an uncommon sign, usually undetected on clinical exams; reported only in a few cases. ${ }^{1-6}$

The underlying mechanisms are still unclear. However, it might be vasospasm of a pontine branch of the basilar artery, primary compression of abducens cranial nerve (VI CN) due to cerebrospinal fluid (CSF) impaired circulation or cisternal prepontine clot. $^{1-3,5,6}$

\section{Description}

We describe two cases of patients whom suffered SAH with bilateral VI CN paresis

\section{Case I}

A 78 year-old woman suffering from hypertension was admitted on the seventh day. Undergoing drowsiness, right moderated hemiparesis and bilateral VI CN paresis and Glasgow Coma Scale (GCS): 10pts. On CT scan was categorized as grade III in Fisher scale and presented diffuse and dense cisternal clot with predominance on the left side. The angiogram showed bilateral carotid siphon aneurysms on posterior communicating artery (PComA) segment and vasospasm (Figure 1 and 2). The left PComA aneurysm was clipped, showing significant clinic evolution. According to the modified Rankin Scale (mRS) on the late sixth month follow up, the patient is graded 2 and fully recovers from the VI CN paresis at the same time.

\section{Case 2}

A 52 year-old man with previous Diabetes type 2 was admitted after a two-day headache, nausea and transitory impairment conscious. At his entrance remained alert, presented bilateral VI CN paresis (Figure 3 ), showed no motor weakness in extremities and shed 15 pts on GCS. Brain CT scan demonstrated SAH Fisher III. CT angiography showed an anterior communicating artery (AComA) aneurysm (Figure 4), obliterated with surgical clipping (Figure 5). The result was significant clinic evolution without recovery from the VI CN paresis at discharge. The mRS shed 1 in the late follow up and fully recovers from the VI $\mathrm{CN}$ paresis on the third month.

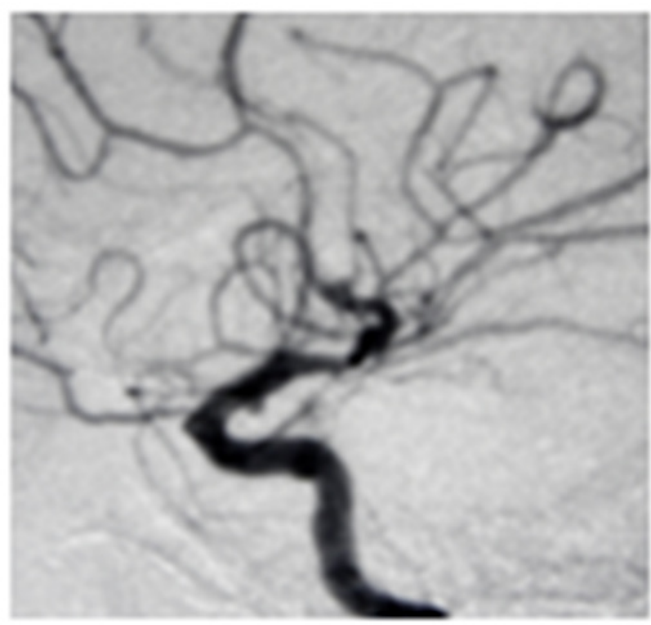

Figure I Left side Pcom Aaneursysm.

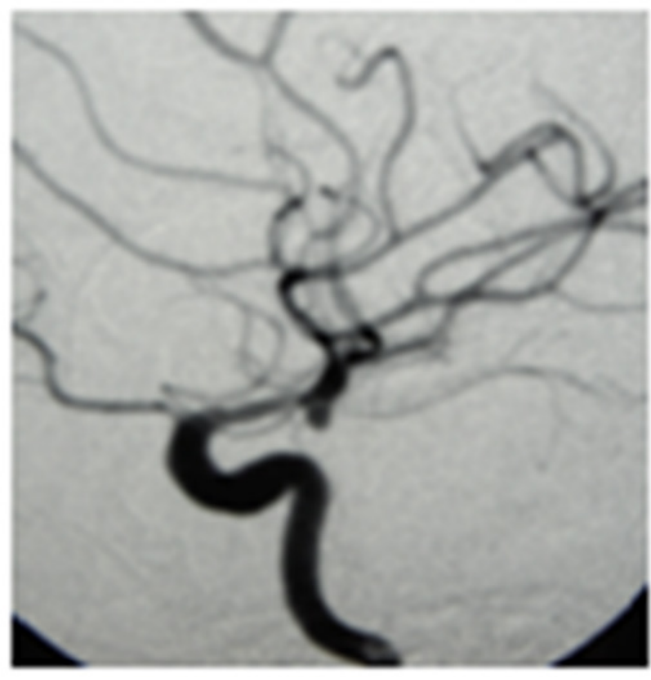

Figure 2 Left side Pcom Aaneursysm. 


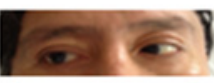

A

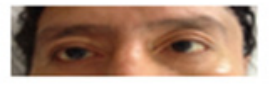

B

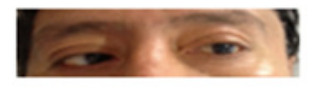

C
Figure 3 Ocular movement examination; A: right, B: front, C: left.

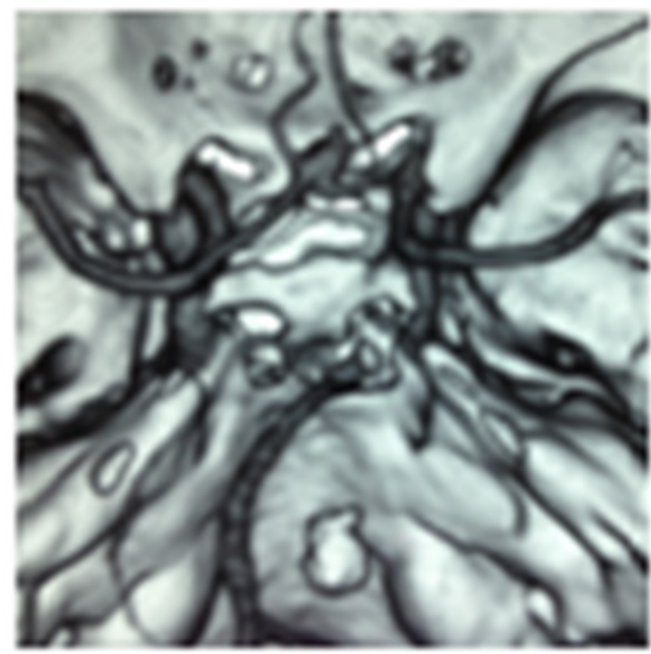

Figure 4 CT angiography demonstrates an AComA aneurysm.

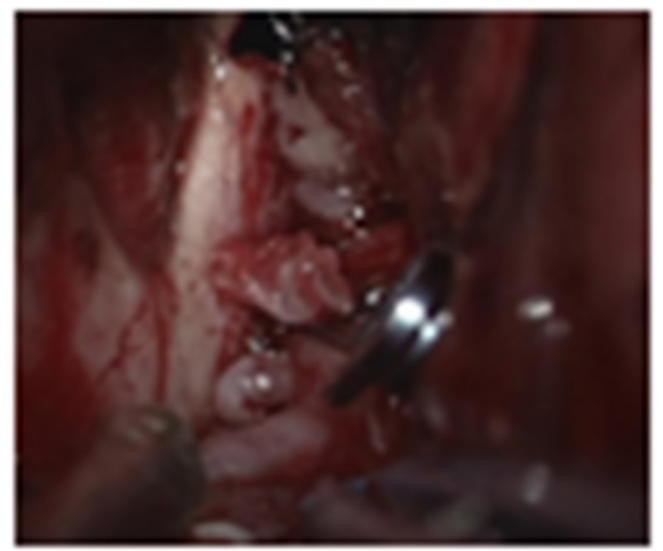

Figure $\mathbf{5}$ Intraoperative photography of the clipped AComA aneurysm.

\section{Discussion}

Causes for abducens nerve paresis are due to many neurosurgical diseases, such as Unruptured Cavernous Carotid Aneurysm, Chronic Intracranial Hypertension and tumors. ${ }^{7,8}$ Tadamichi found an organic lesion on the sixth nerve dysfunction, especially between 20 and 49 year-olds. $^{7}$ Aneurysms constitute only between 2 to $3.6 \%$ of the cases. $^{7-10}$

The reported incidence after SHA varies according to the authors. For example, Laun \& Tonn ${ }^{4}$ analyzed the dysfunctions of cranial nerves after SHA with abducens nerve palsy as the second most frequent - only 3.3\% and Munakata et al. ${ }^{5}$ found $5.9 \%$. The aneurysmal locations reported in previous studies are found in the vertebral artery, basilar artery, PICA, carotid siphon in the PComA segment, AComA and middle cerebral artery. ${ }^{4,-10}$

Theories on the origin of abducens nerve dysfunction after SHA are vasospasm of a pontine branch of the basilar artery and primary compression of abducens cranial nerve, caused by cerebrospinal fluid impaired circulation or cisternalprepontine clot and the previous series have suggested various conclusions. Nathal et al. ${ }^{2}$ in one of his first reports, and Göksu et al. ${ }^{1}$ analyzed cases of ruptured ACoA aneurysm, concluding direct compression by blood or CSF in the subarachnoid space is the most important physiopathologic mechanism. Munakata et al. ${ }^{5}$ compared two groups of patients, with and without abducens nerve dysfunction. He analyzed the thickness of the prepontine subarachnoidal clot in both groups. He found statistically significant differences; leading to the conclusion that prepontine clot seemed to be the main factor. The most recent publication by Jeon et al. ${ }^{6}$ reported a slower recovery of abducens nerve after embolization of ruptured ACoA aneurysm, followed by lumbar cerebrospinal fluid drainage (during 6 days), indicating CSF entrapment as the dominant factor.

We assume cisternal prepontine clot and CSF impaired circulation with mechanical compression on the VI $\mathrm{CN}$ are the predominant factors in our cases, instead vasospasm; given the lack of facial nerve alteration. Both patients presented the alteration at the time of admission and brain CT controls $24 \mathrm{hs}$ after surgery did not show signs of prepontine clot. Therefore, the speculation on CSF circulation impairment has an added factor in the long-term. Further studies with more population may reveal more precisely which is the main factor.

Recovery time is reported to last 3 to 9 weeks and 3 to 6 months after bleeding., ${ }^{3,11}$ Ziyal et al. ${ }^{3}$ indicates shorter periods due to fenestration of Liliequist membrane, as well as CSF circulation improves in the prepontine cistern. Despite we did not performed this surgical maneuver on these cases, the first one reached a full recovery at 6 months and the second one at 3 months.

\section{Conclusion}

Compromise of the abducens nerve after aneurysmal subarachnoid hemorrhage might be linked to the presence of a thick clot in the prepontine cistern and CSF impaired circulation. Patients usually show significant recovery after treatment, within 3 to 6 months.

\section{Acknowledgements}

None.

\section{Conflicts of interest}

None.

\section{References}

1. Göksu E, Akyüz M, Gürkanlar D, et al. Bilateral abducens nerve palsy following ruptured anterior communicating artery aneurysm: report of 2 cases. Neurocirugia (Astur). 2007;18(5):420-422.

2. Nathal E, Yasui N, Suzuki A, et al. Ruptured anterior communicating artery aneurysm causing bilateral abducens nerve paralyses-case report. Neurol Med Chir (Tokyo). 1992;32(1):17-20.

3. Ziyal IM, Ozcan OE, Deniz E, et al. Early improvement of bilateral abducens nerve palsies following surgery of an anterior communicating artery aneurysm. ActaNeurochir (Wien). 2003;145(2):159-161.

4. Laun A, Tonn JC. Cranial nerve lesions following subarachnoid hemorrhage and aneurysm of the circle of Willis. Neurosurg Rev. 1988;11(2):137-141.

5. Munakata A, Ohkuma $\mathrm{H}$, Nakano $\mathrm{T}$, et al. Abducens nerve pareses associated with aneurysmal subarachnoid hemorrhage. Cerebrovasc Dis. 2007;24(6):516-519.

6. Jeon JS, Lee SH, Son YJ, et al. Slowly recovering isolated bilateral abducens nerve palsy after embolization of ruptured anterior communicating artery aneurysm. $J$ Korean Neurosurg Soc. 2013;53(2):112-114. 
7. Akagi T, Miyamoto K, Kashii S, et al. Cause and prognosis of neurologically isolated third, fourth, or sixth cranial nerve dysfunction in cases of oculomotor palsy. Jpn J Ophthalmol. 2008;52(1):32-35.

8. Bendszus M, Beck A, Koltzenburg M, et al. MRI in isolated sixth nerve palsies. Neuroradiology. 2001;43(9):742-745.

9. Patel SV, Mutyala S, Leske DA, et al. Incidence, associations, and evaluation of sixth nerve palsy using a population-based method. Ophthalmology. 2004;111(2):369-375
10. Rush JA, Younge BR. Paralysis of cranial nerves III, IV, and VI. Cause and prognosis in 1,000 cases. Arch Ophthalmol. 1981;99(1):76-79.

11. Garg K, Singh PK, Mahapatra AK, et al. Bilateral abducens nerve palsy associated with subarachnoid hemorrhage. $\mathrm{Br} J$ Neurosurg. 2014;28(6):771-775. 